\title{
NMR Relaxometry to Characterize the Drug Structural Phase in a Porous Construct
}

\section{Authors: Linn W. Thrane, Emily A. Berglund, James N. Wilking, David Vodak, and Joseph D. Seymour}

This document is the unedited author's version of a Submitted Work that was subsequently accepted for publication in Molecular Pharmaceutics, copyright ( $)$ American Chemical Society after peer review. To access the final edited and published work, see https://doi.org/10.1021/ acs.molpharmaceut.8b00144.

Thrane, Linn W., Emily A. Berglund, James N. Wilking, David Vodak, and Joseph D. Seymour, "NMR Relaxometry to Characterize the Drug Structural Phase in a Porous Construct," Molecular Pharmaceutics, June 2018; 15(7): 2614-2620. doi: 10.1021/acs.molpharmaceut.8b00144 


\title{
NMR Relaxometry to Characterize the Drug Structural Phase in a Porous Construct
}

\author{
Linn W. Thrane, ${ }^{\dagger, \ddagger}$ Emily A. Berglund, ${ }^{\dagger}$ James N. Wilking, ${ }^{\dagger}$ David Vodak, ${ }^{\S}$ and Joseph D. Seymour*,† \\ ${ }^{\dagger}$ Department of Chemical and Biological Engineering, Montana State University, Bozeman, Montana 59717-3920, United States \\ ${ }^{\ddagger}$ Department of Mechanical and Industrial Engineering, Montana State University, Bozeman, Montana 59717, United States \\ ${ }^{\S}$ Bend Research Incorporated, Lonza, Bend, Oregon 97701, United States
}
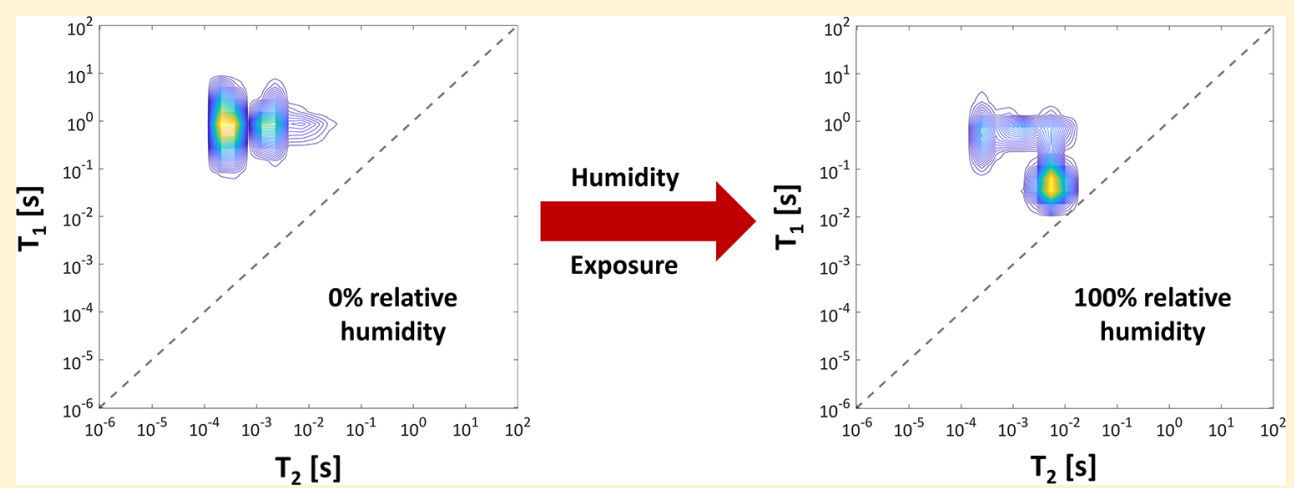

ABSTRACT: Nuclear magnetic resonance (NMR) frequency spectra and $T_{2}$ relaxation time measurements, using a high-power radio frequency probe, are shown to characterize the presence of an amorphous drug in a porous silica construct. The results indicate the ability of non-solid-state NMR methods to characterize crystalline and amorphous solid structural phases in drugs. Two-dimensional $T_{1}-T_{2}$ magnetic relaxation time correlation experiments are shown to monitor the impact of relative humidity on the drug in a porous silica tablet.

KEYWORDS: NMR relaxometery, porous construct, amorphous, crystalline, melt solidification

\section{INTRODUCTION}

Drug solubility depends on whether the drug is in the crystalline or amorphous solid state. ${ }^{1}$ Methods such as spray drying $^{2}$ and pore entrapment ${ }^{3-6}$ are well established approaches to impact molecular dynamics during solidification and control the drug structural phase. Nuclear magnetic resonance (NMR) methods have been applied to characterize the amorphous and crystal structures of pharmaceuticals using solid-state (ssNMR) methods. ${ }^{7-10}$ These ssNMR methods require sample spinning and typically grinding of the sample into a powder, and the incompatibility of the methods with the liquid state precludes observing transitions from the liquid melt to the solid state during solidification. Liquid-state NMR relaxometry is well established to characterize pore size distributions in porous media. ${ }^{11,12}$ NMR relaxation times are sensitive to phase transitions from the liquid to the solid state. $^{13,14}$ Somewhat analogous to the solidification processes studied here is NMR cryoporometry in porous media, which relies on the Gibbs-Thomson effect and the presence of nanoscale unfrozen water layers at the boundary of the solid porous matrix and the ice crystals in the bulk pores to characterize porous systems. $^{15,16}$

In this article, we describe the use of a purpose built highpower radio frequency (rf) pulse probe to measure the solidification of the drug fenofibrate in bulk and within a porous matrix. The probe allows for high-power short duration rf pulses capable of acquiring signal from the solid-state drug without sample spinning. NMR spectra indicate amorphous domains after solidification in the porous matrix that are not present in the bulk solidification, assisting in the verification and interpretation of the novel relaxation data presented. The impact of relative humidity $(\mathrm{RH})$ on the structure of the solidstate drug in the porous matrix is shown to correlate with NMR relaxation behavior. The results quantify the impact of porous matrix restriction on the drug solidification and indicate the potential for quality control and process monitoring by NMR.

\section{THEORY}

NMR frequency spectra and magnetic relaxation times are sensitive to the liquid or solid phase of the sample and the solid-state structural crystal or amorphous phase. The frequency spectra of liquids are Lorentzian due to the exponential form of the time-dependent free induction decay (FID) voltage signal, while those of solids are Gaussian. ${ }^{17,18}$ This is due to motional averaging of the dipolar coupling of the 
${ }^{1} \mathrm{H}$ protons in liquids by rotational diffusion. ${ }^{17}$ In solids, the correlation time of the dipolar coupling is much longer and results in a Gaussian FID that decays away rapidly in time $(\sim 1-100 \mu \mathrm{s})$, generating a broad Gaussian line width in the frequency domain. ${ }^{18}$ The spin-spin $T_{2}$ relaxation time depends on the proton dipolar coupling and is short $(<1 \mathrm{~ms})$ for solids and longer $(>100 \mathrm{~ms})$ for liquids, and it determines the line shape and line width. The solid phase ordering in crystalline phases increases the dipolar coupling with corresponding fast in time Gaussian FID decays with a $T_{2}$ shorter than that in amorphous solids, where disorder and slightly increased mobility generate longer $T_{2}$ and potentially shorter time Gaussian and longer time exponential FIDs. ${ }^{12,19}$ The spinlattice $T_{1}$ relaxation in liquids is at the order of seconds, and $T_{1}$ $\sim T_{2}$, while in solids, $T_{1}$ is much longer than $T_{2}{ }^{12}$ The solid phase $T_{1}$ in more ordered crystalline states is longer than that in amorphous states.

\section{METHODS}

The porous matrix used in these experiments was a porous tablet made from colloidal silica. Silica with a stable-ordered mesoporous structure has been shown to be a promising controlled drug delivery system. ${ }^{3}$ Charge-stabilized, amorphous colloidal silica (Nissan Chemical, MP-1040-H) suspended in water with an average particle diameter of $d=100 \mathrm{~nm}$ and low size polydispersity $(<5 \%)$ was used for all of the experiments. The surface of the colloidal silica is negatively charged with a surface charge density between 8 and $10 \mathrm{~nm}^{-1}$ and free of residual organics, as stated by the manufacturer. Colloidal silica (300 $\mu \mathrm{L}$ ) was added to cylindrical molds and left to solidify for $24 \mathrm{~h}$. The tablet dimensions were set by the dimensions of the mold used during the slipcasting process. Tablets were cylindrical in shape, with a diameter and height on the order of $0.5-1 \mathrm{~cm}$. Silica particles were packed into a random structure, as revealed by small angle neutron scattering experiments. The characteristic pore size could be estimated by calculating the void created by three kissing spheres: $D=$ $0.15 d$; with $d$ estimated as $d \approx 15 \mathrm{~nm}$. After $24 \mathrm{~h}$, the fenofibrate was heated to $10{ }^{\circ} \mathrm{C}$ above its melting point of 81.1 ${ }^{\circ} \mathrm{C}$, and the drug was loaded into the tablet by imbibing the drug in liquid form. The fenofibrate was absorbed into the tablet by capillary action. Once the imbibition process was complete, the mass fraction of the drug in the composite was $0.18 \pm 0.01$, as measured using thermogravimetric analysis. This corresponds to the silica colloid volume fraction of $\varphi=$ 0.71 , based on the density of amorphous silica, $\rho_{\text {silica }}=2.196 \mathrm{~g} /$ $\mathrm{cm}^{3}$, and the density of fenofibrate, $\rho_{\text {fenofibrate }}=1.18 \mathrm{~g} / \mathrm{cm}^{3}$. Measurements indicate that the void space was filled completely by the drug during imbibition. Finally, the tablet was wiped down to remove any excess drug on the surface before it was placed in a $5 \mathrm{~mm}$ NMR tube. The bulk drug sample was prepared by adding the powdered drug to a $5 \mathrm{~mm}$ NMR tube and melting the drug in the tube. The sample was quickly placed in a Bruker $250 \mathrm{MHz}$ superconducting magnet integrated to an Avance III spectrometer, where frequency spectra and $T_{2}$ measurements were performed using a highpower $\mathrm{rf}$ probe with a $5 \mathrm{~mm}$ rf coil custom built by Bruker. The temperature was controlled at $20{ }^{\circ} \mathrm{C}$ using the Bruker BTU system with $\mathrm{N}_{2}$ gas flow. The $T_{2}$ measurements were executed using the standard Carr-Purcell-Meiboom-Gill (CPMG) pulse sequence with an echo time of $\tau_{\mathrm{E}}=12 \mu \mathrm{s}, 500-4000$ echoes, depending on the sample, acquisition sampling dwell time of $1 \mu \mathrm{s}$, and $7.5 \mu \mathrm{s}$ rf pulses at $100 \mathrm{~W}$ power. The signal was acquired using 4 averages of a 16-phase cycle acquisition. The rapid data acquisition in the short $2 \cdot \tau_{\mathrm{E}}$ period precluded full acquisition of the echo, so the data were not encoded for spectral frequency shift. Experiments with $\tau_{\mathrm{E}}$ varying from 12 to $400 \mu$ s indicated no $T_{2}$ dispersion effect due to potential spin locking of longer $T_{2}$ components with rotating frame spinlattice relaxation times $T_{1 \rho}$ of similar magnitude. ${ }^{10,20}$ The $T_{2}$ relaxation data were converted by inverse Laplace transformation to a distribution of relaxation populations. ${ }^{11,19,21}$

To explore the impact of $\mathrm{RH}$ on the solidified drug with time, tablets were stored in an environment with controlled $\mathrm{RH}$ at $T=20{ }^{\circ} \mathrm{C}$. The $0 \% \mathrm{RH}$ conditions were set by sealing the tablet in a container with an excess of dry desiccant (Drierite). The $100 \% \mathrm{RH}$ conditions were set by sealing the composites in a container with excess liquid water. Liquid water was not allowed to be in contact with the tablet, but it created a saturated vapor pressure at equilibrium in an enclosed environment. Measurements of a tablet kept in a $0 \% \mathrm{RH}$ environment were compared to the measurements of a tablet kept in a $100 \% \mathrm{RH}$ environment for 2 weeks. $T_{1}$ and $T_{2}$ relaxation data are acquired by performing $1 \mathrm{D} T_{2}$ experiments and $2 \mathrm{D} T_{1}-T_{2}$ correlation experiments. The $T_{1}-T_{2}$ measurements are performed using an inversion recovery combined with a CPMG pulse sequence. ${ }^{11}$ The same parameters used in the $T_{2}$ measurements were used for the CPMG portion of the $T_{1}-T_{2}$ measurement, except that 2 averages of the 16-phase cycle acquisition were used. Inversion recovery was performed with inversion times logarithmically spaced from $1 \mathrm{~ms}$ to $50 \mathrm{~s}$. Inverse Laplace transforms of the relaxation data were performed in $1 \mathrm{D}$ for $T_{2}$ and $2 \mathrm{D}$ for $T_{1}-T_{2}$ experiments to provide distributions of relaxation times. Data were collected on day $1,3,7$, and 14 of the storage in $100 \%$ RH. To avoid exposing the sample to lower $\mathrm{RH}$ during measurements, four samples were prepared so that none of the tablets were removed from the high $\mathrm{RH}$ environment until the measurement was performed.

\section{RESULTS AND DISCUSSION}

Drug Solidification. Once the tablet or the bulk drug is placed in the magnet, frequency spectra and $T_{2}$ measurements are interleaved and performed continuously until the drug is fully solidified and no further changes are observed. The fenofibrate is originally in powder form of ground crystal but takes on a more ordered crystalline structure when it solidifies after being melted. ${ }^{22}$ This is indicated by the $T_{2}$ measurements of the drug in powder form and after the melted drug has solidified.

The $T_{2}$ distributions in Figure 1 show that the drug has two $T_{2}$ populations before and after being melted, and the signal amplitude distribution between the two peaks remains approximately the same. A shift toward shorter $T_{2}$ relaxation times is observed in the melted and solidified drug. Additionally, the total signal amplitude of the solidified drug is 2 orders of magnitude lower than that of the powdered drug; however, this cannot be seen in Figure 1 due to both of the $T_{2}$ distributions being normalized by the population amplitude maximum for visualization of the shift in the $T_{2}$ relaxation time. The decrease in the total measured signal amplitude in the recrystallized system is due to the $T_{2}$ relaxation times of the drug becoming too short, i.e., less than a $\tau_{\mathrm{E}}$ of $12 \mu \mathrm{s}$, for the NMR pulse sequence to detect, indicating a more ordered crystalline structure than the powder form. The two populations thus represent the most rotationally mobile 


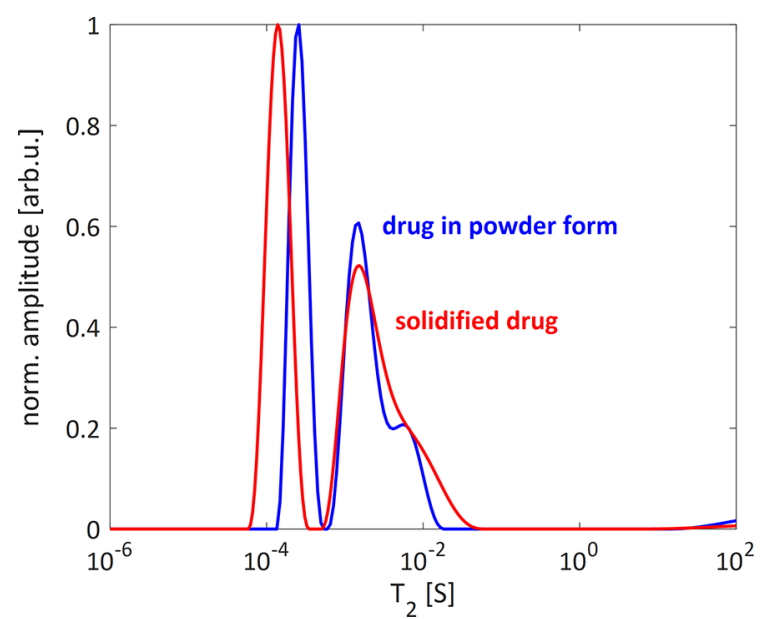

Figure 1. $T_{2}$ distributions of the drug in powder form (blue/black) and the solidified drug after being melted and recrystallized (red/ gray). Two populations are observed in both samples, and the signal intensity ratio between the two populations remains approximately the same before and after melting. The population with the shortest $T_{2}$ time experiences a shift toward shorter relaxation times when solidifying after being melted.

protons in the system with $T_{2}>\sim 50 \mu \mathrm{s}$. The populations cannot be assigned to specific moieties on the molecule. However, from spectroscopic experiments and quantum mechanical simulations, the crystal structure indicates stacking of benzene rings. ${ }^{22}$ The population at $T_{2} \sim 10^{-4} \mathrm{~s}$ shifts to lower $T_{2}$ values in the melted solidified sample relative to the powdered form. It is expected that the melt process will allow reorientation of molecules for a smaller molecular spacing in the crystal, and protons in this population indicate that effect. The more rotationally mobile protons at $T_{2} \sim 1 \times 10^{-3} \mathrm{~s}$ are associated with the methyl groups furthest from the stacked benzene rings, and the broad distribution of $T_{2}$ values indicates that they experience varying rotational mobility depending on the exact location on the molecule, which is consistent with the model of Heinz et. al. ${ }^{22}$

The bulk drug took approximately $30 \mathrm{~min}$ to fully solidify after melting, while it took 3-4 h for the drug in the porous tablet to solidify. To ensure no further changes occurred in the tablet past the first $4 \mathrm{~h}$, the sample was stored, and additional measurements were performed after $21 \mathrm{~h}$. Figure $2 \mathrm{a}$ shows the frequency spectra of the drug in bulk in the liquid melt state at 0 and in the solid state at $27 \mathrm{~min}$. The melt state signal is a convolution of a Gaussian and Lorentzian decay. This indicates the high degree of molecular order, likely due to $\pi$ stacking of the benzene rings in the molten state. The Lorentzian portion of the frequency spectrum at $0 \mathrm{~min}$ is liquid-like, where the molecular mobility, i.e., the random modulation of the dipolar coupling, is fast. In bulk, the drug solidifies with unrestricted molecular mobility, and the final result is a highly ordered crystalline structure. The slow random modulation of the dipolar coupling produces a pure Gaussian line shape in the frequency spectrum, as can be seen in Figure $2 \mathrm{a}$ at $27 \mathrm{~min}^{18}$

A similar Lorentzian distribution is observed in the frequency spectra for the drug in a porous tablet in the liquid melt state at 0 min (Figure 2b). However, in addition to the broad Gaussian distribution seen in the pure drug, a remaining Lorentzian distribution can be seen at $21 \mathrm{~h}$ for this sample. The combination of Gaussian and Lorentzian line shapes in the frequency spectrum suggests that when restricted in a porous media, some of the drug will not reach the highly ordered crystalline structure of the pure drug. The Gaussian line shape confirms that the drug has solidified, but the remaining Lorentzian line shape indicates the presence of a highly amorphous state of the solidified drug or supercooled liquid. $^{23,24}$

When the bulk drug solidifies, a highly ordered bulk crystal structure forms. In a confined environment of $15 \mathrm{~nm}$ pores, as used here, fenofibrate has been shown to produce nanocrystals by ${ }^{13} \mathrm{C}$ MAS ssNMR and XRPD. ${ }^{25}$ In that study, ssNMR data showed line broadening with the pore size decreasing from 300 to $20 \mathrm{~nm}$, which was attributed to the surface disorder as nanocrystals became small, thus differentiating the surface drug versus nanocrystalline core drug. ${ }^{25}$ At $12 \mathrm{~nm}$ pore size, the ssNMR still indicated crystalline drug, but the ${ }^{13} \mathrm{C}$ spectra broadening and XRPD data indicated loss of long-range order. WAXS diffraction on the bulk fenofibrate and fenofibrate in the porous construct for our samples shows a behavior similar to the XRPD data of reference, ${ }^{25}$ with crystalline behavior in bulk and a large background silica signal in the small pore tablet (Figure 3). The line width of the Gaussian portion of the spectra for the bulk and tablet-solidified samples (Figure 2) is similar, indicating the presence of nanocrystals of drug in the
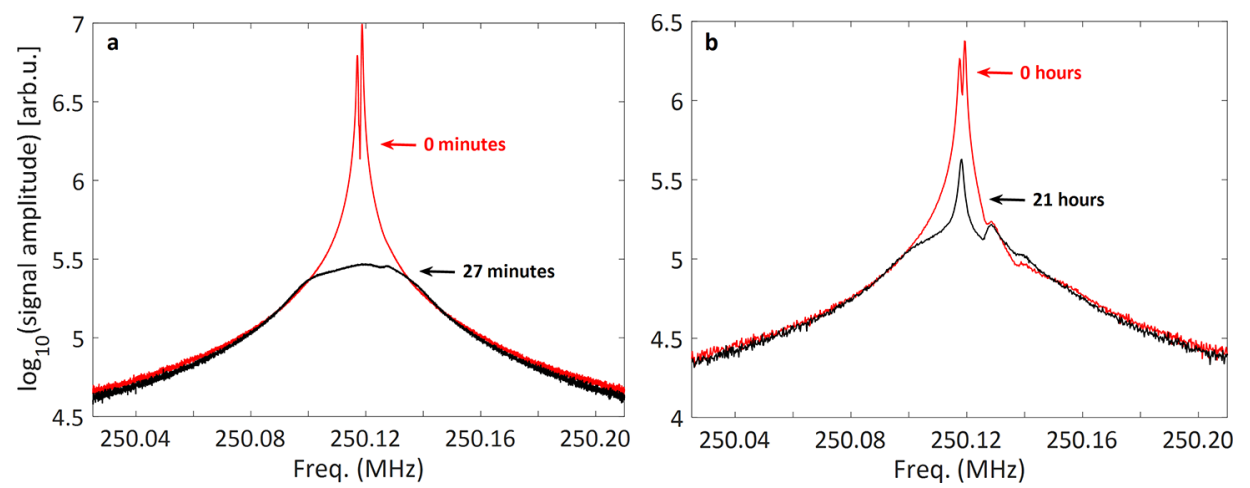

Figure 2. Frequency spectra of drug solidifying in (a) bulk and (b) tablet. In both samples, the drug starts out (red/gray) as highly amorphous, as indicated by the Lorentzian shape of the frequency spectra. Once the solidification process is complete, the pure drug has solidified (black) to a highly ordered crystalline structure, resulting in a broad Gaussian frequency peak. The final frequency spectrum for the drug in the tablet is a combination of a broad Gaussian distribution and a Lorentzian, indicating that the drug solidifying in the restricted environment of the porous matrix is more amorphous than that of the bulk sample. 


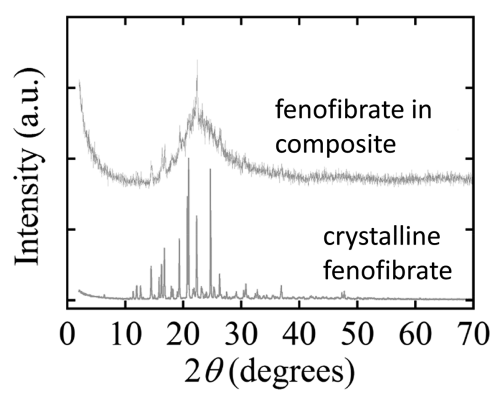

Figure 3. Wide-angle X-ray (WAXS) diffraction spectra for the fenofibrate $-\mathrm{SiO}_{2}$ composite with unprocessed crystalline fenofibrate powder direct from the manufacturer. The primary Bragg peaks in the composite scattering do not match up with those in the crystalline active, indicating they do not have the same crystal structure. A broad amorphous $\mathrm{SiO}_{2}$ peak dominates the composite sample signal.

pore. The Lorentzian component indicates an amorphous component due to the impact of the silica-fenofibrate surface interactions. This could be due to the supercooled liquid or amorphous surface solid. Given the rapid crystallization behavior of supercooled fenofibrate, which occurs on the time scale of minutes in solution in the presence of a nucleation site, $^{23,24}$ we believe it is a surface-impacted solid. ${ }^{25}$

The $T_{2}$ data collected during the solidification process (Figure 4) indicate that a majority of the signal originates from the population with $T_{2}$ relaxation times of $\sim 10^{-3} \mathrm{~s}, 9.9 \times 10^{-4} \mathrm{~s}$ in the bulk fenofibrate and $9.3 \times 10^{-4} \mathrm{~s}$ in the tablet, when the drug is in liquid form. Note that the most rotationally mobile protons in the melt at $T_{2} \sim 10^{-2} \mathrm{~s}$ are no longer fully resolved in the solidifying sample. These most mobile protons associated with the methyl protons become a shoulder on the $T_{2} \sim 10^{-3} \mathrm{~s}$ population as the crystal forms. In both systems, the signal amplitude of the $\sim 10^{-3} \mathrm{~s} T_{2}$ time population decreases significantly, and the signal amplitude of the shorter $T_{2}$ time population, $\sim 10^{-4} \mathrm{~s}$, increases during the drug solidification. These results indicate that the relaxation time of a portion of the fenofibrate shifts from the decreasing long $T_{2}$ population to the short $T_{2}$ population, and the remaining drug exhibits $T_{2}$ times too short to detect with the $12 \mu \mathrm{s} \tau_{\mathrm{E}}$. In the bulk fenofibrate (Figure 4a), the signal amplitude of the $\sim 10^{-3} \mathrm{~s}_{2}$ population keeps decreasing until a greater portion of the remaining signal originates from the $\sim 10^{-4} \mathrm{~s} T_{2}$ population. For the fenofibrate in the tablet (Figure $4 \mathrm{~b}$ ), the signal amplitude of the $\sim 10^{-3} \mathrm{~s} T_{2}$ population stops decreasing when the remaining signal is evenly distributed between the two populations. The even distribution of signal amplitude in the final state of fenofibrate in the porous medium compared to that of the bulk fenofibrate confirms the results from the frequency spectra that the drug takes on a more amorphous structure when solidifying within a restricted environment. ${ }^{25}$

Impact of Relative Humidity. The impact of $\mathrm{RH}$ on the structure of a solid-state drug is relevant to long time storage of the drug. If stored in a high $\mathrm{RH}$ environment over time, water will begin to inhabit the pore space of the tablet due to capillary condensation. $T_{1}-T_{2}$ correlation measurements are used to quantify the effect of $100 \% \mathrm{RH}$ on fenofibrate confined in a porous matrix over a 2 week period. For comparison, $T_{1}-T_{2}$ data were collected from fenofibrate in a tablet stored at $0 \%$ $\mathrm{RH}$. At $0 \% \mathrm{RH}$, the $T_{1}-T_{2}$ correlation data (Figure 5) reveal two main populations with identical $T_{1}$ relaxation times and $T_{2}$ times at the order of $10^{-4}$ and $10^{-3} \mathrm{~s}$ and the broad shoulder out to $10^{-2} \mathrm{~s}$ on the $10^{-3} \mathrm{~s}$ population. These are similar $T_{2}$ times to those observed in the $T_{2}$ distributions in Figures 1 and 4. The tablet stored at $0 \% \mathrm{RH}$ showed no significant changes over time.

$T_{1}-T_{2}$ measurements from day 1 of a tablet stored at $100 \%$ $\mathrm{RH}$ reveal a third population in the $T_{1}-T_{2}$ correlation map closer to the parity line (gray dashed line, Figure 6a), representing $T_{1}=T_{2}$. Populations located on or close to the parity line are representative of a liquid phase, and it can therefore be assumed that this third population is water that has entered the porous matrix of the tablet as a result of its high $\mathrm{RH}$ environment. The signal amplitude of the population representing water increases with time and is approximately 1 order of magnitude greater by day 14 . The water population is also shifting toward longer $T_{1}$ and $T_{2}$ relaxation times, with $T_{1}$ increasing from $10^{-2} \mathrm{~s}$ on day 1 to $10^{-1} \mathrm{~s}$ by day 14 , and $T_{2}$ increasing from $10^{-3}$ to $10^{-2} \mathrm{~s}$ by day 14 . The increase in relaxation time along with the increase in signal amplitude of the water population indicate that the water is progressively occupying more of the pore space as well as larger pores in the
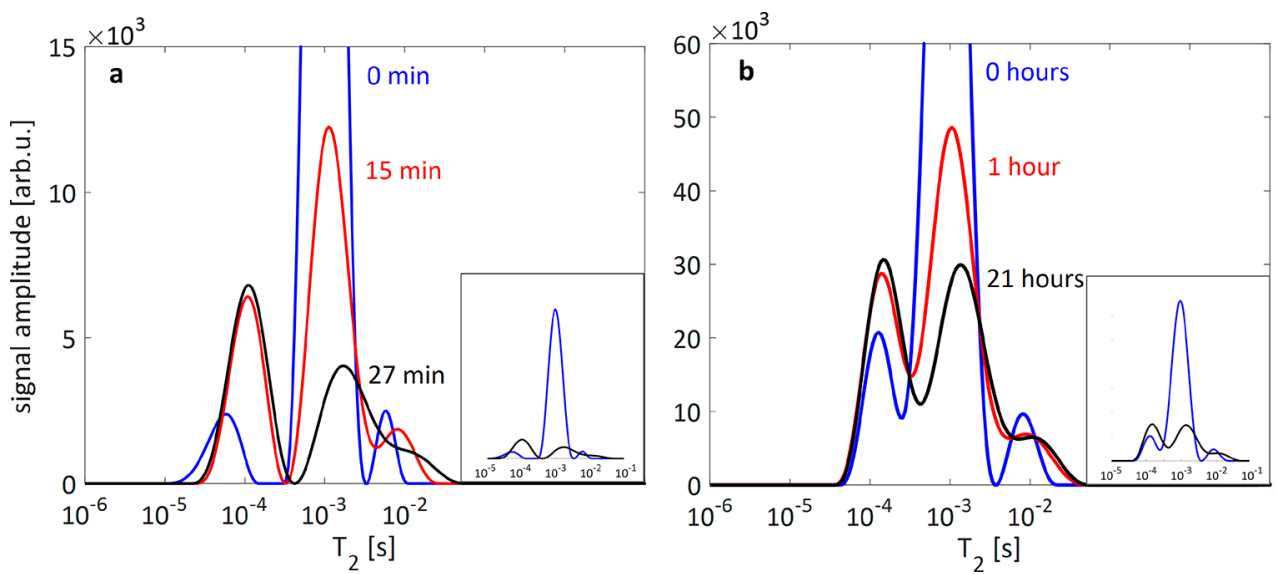

Figure 4. $T_{2}$ distributions of drug solidifying in (a) bulk and (b) tablet. The full signal amplitude is shown in the right corner of the plots. There is a large signal loss in both samples due to the solidification of the drug. Once solidification is complete in the bulk sample, most of the signal from the longer $T_{2}$ population, $\sim 10^{-3} \mathrm{~s}$, is lost, and most of the signal results from the shorter $T_{2}$ population, $\sim 10^{-4} \mathrm{~s}$. In the tablet sample, there is an equal signal distribution between the $\sim 10^{-3} \mathrm{~s}$ and the $\sim 10^{-4} \mathrm{~s} T_{2}$ populations when the solidification is complete, confirming the results seen in the frequency spectra suggesting that the drug in the tablet is in a more amorphous mobile state than that of the highly ordered bulk sample. 


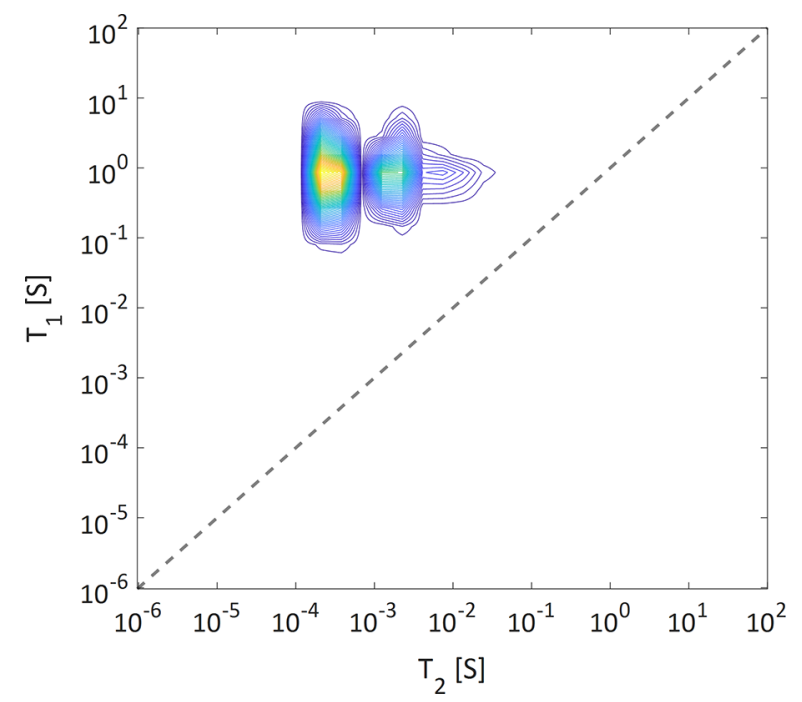

Figure 5. $T_{1}-T_{2}$ correlation data from the model drug in a tablet kept at $0 \% \mathrm{RH}$. The $T_{1}-T_{2}$ data indicate that there are two populations with different $T_{2}$ relaxation times present in the tablet at $0 \% \mathrm{RH}$. The two populations have identical $T_{1}$ relaxation times and $T_{2}$ times on the order of $10^{-4}$ and $10^{-3} \mathrm{~s}$. porous matrix over the 14 day period. The two populations representing the drug in the tablet decrease in amplitude over the 14 day period but do not experience a significant shift in $T_{1}$ or $T_{2}$ relaxation time.

This is more readily observed in Figure 7, where the 1D $T_{2}$ distributions from day $1,3,7$, and 14 are presented. The $T_{2}$ distributions are normalized to the maximum population amplitude recorded on day 14 so that the growth of the water peak can be visualized. The population with highest signal amplitude in the $T_{2}$ distribution for day 1 (Figure $7 \mathrm{a}$ ) represents the signal originating from both the highly restricted mobility water population and the drug population with $10^{-3} \mathrm{~s}$ $T_{2}$ from Figure 6a. On day 1, these two populations exhibit similar $T_{2}$ times and can therefore not be distinguished in the $T_{2}$ distribution. On day 3 , the water population has experienced a large enough increase in $T_{2}$ times to appear as a third peak with a $T_{2}$ of $5.6 \times 10^{-3} \mathrm{~s}$ in Figure $7 \mathrm{~b}$. This very short $T_{2}$ time indicates water in highly restricted thin films. The water peak keeps increasing in signal amplitude and shifting toward longer $T_{2}$ times until a $T_{2}$ of $2.3 \times 10^{-2} \mathrm{~s}$ is reached on day 14 (Figure $7 \mathrm{~d}$ ). This $T_{2}$ time indicates highly restricted water relative to bulk, but the near order of magnitude increase from day 3 suggests significant changes in the restriction length scale. The two populations representing the drug have experienced a slight decrease in signal amplitude by day 14 but no significant shift in
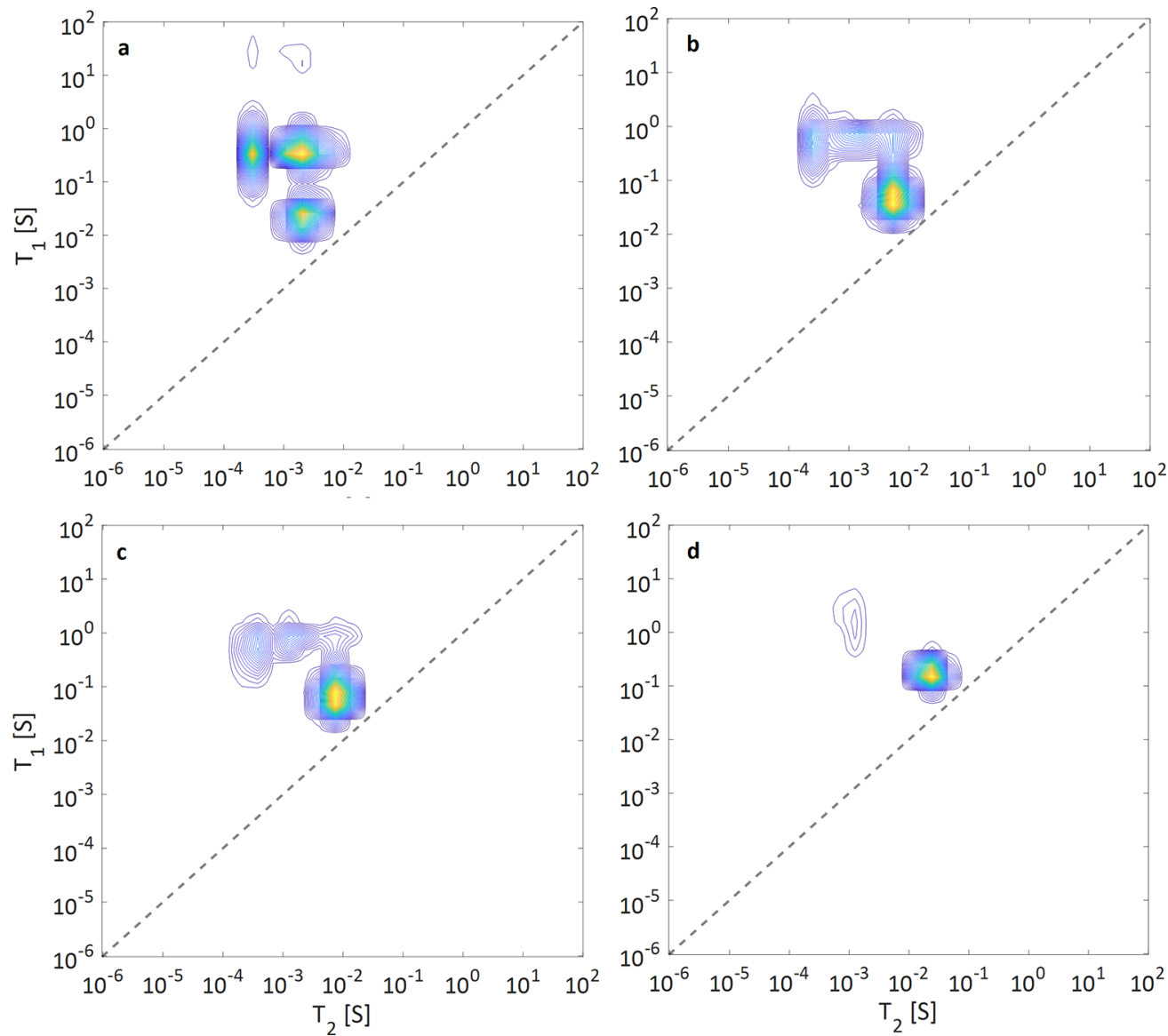

Figure 6. $T_{1}-T_{2}$ correlation data of drug in a tablet stored at $100 \% \mathrm{RH}$ for (a) 1 , (b) 3 , (c) 7 , and (d) 14 days. It can be seen already on day 1 that a third population has appeared closer to the $T_{1}=T_{2}$ parity line (gray dashed line) when compared to the $T_{1}-T_{2}$ data at $0 \% \mathrm{RH}$. This population is in liquid form and is attributed to water building up in the porous matrix of the tablet. The peak representing water is increasing in amplitude with time and is approximately 1 order of magnitude greater by day 14 . The two populations representing the drug trapped within the pore space of the tablet are decreasing in amplitude with time. 

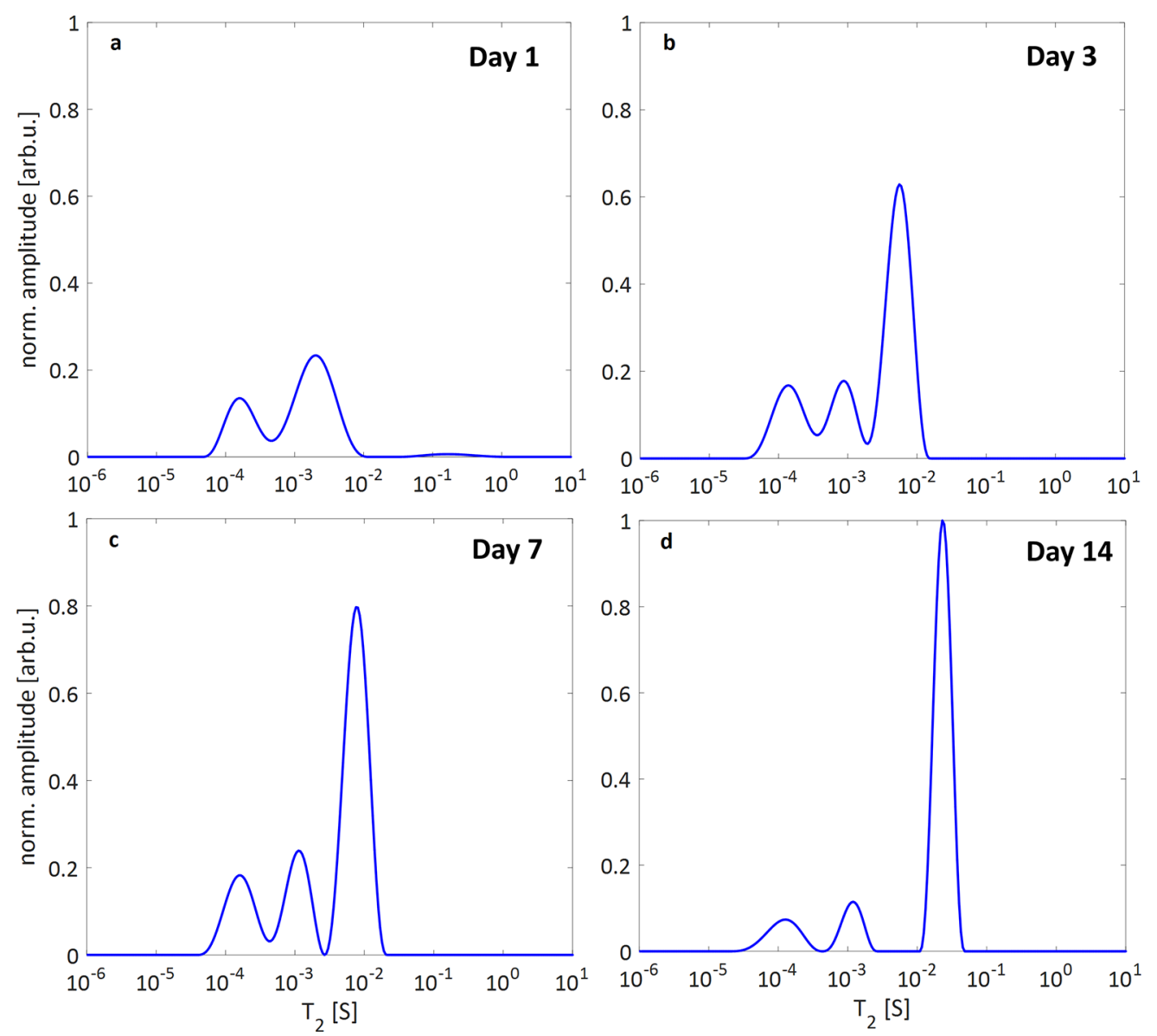

Figure 7. $T_{2}$ distributions of the drug in a tablet stored at $100 \% \mathrm{RH}$ for (a) 1 day, (b) 3 days, (c) 7 days, and (d) 14 days. On day 1 (a), the water population and the drug population with the longest $T_{2}$ time, $\sim 10^{-3} \mathrm{~s}$, overlap and appear as just one population. By day 3 (b), the relaxation time of the water population increased, and the water population appears as the third peak with the longest $T_{2}$ time at $5.6 \times 10^{-3} \mathrm{~s}$ in the $T_{2}$ distribution. The water peak continues to shift toward longer relaxation times, $7.7 \times 10^{-3} \mathrm{~s}$ on day 7 and $2.3 \times 10^{-2} \mathrm{~s}$ on day 14 , as well as increasing in signal amplitude.

$T_{2}$ times, indicating drug insolubility. Since the drug appears insoluble and the water occupies larger length scale environments, the pore structure must be altered by the water through tablet volume expansion or breakup. This effect is also observed visually.

\section{CONCLUSION}

NMR frequency spectra and 1D $T_{2}$ measurements were used to characterize the solidification of fenofibrate in bulk and in a porous matrix. The results indicate that fenofibrate takes on an amorphous structure in a confined environment of $\sim 15 \mathrm{~nm}$ compared to that of the highly ordered crystal structure formed by the bulk drug, and they confirm the application of non-solidstate NMR to distinguish and characterize crystalline and amorphous solid structural phases in drugs. One-dimensional $T_{2}$ and $2 \mathrm{D} T_{1}-T_{2}$ relaxation correlation experiments were used to investigate the impact of $\mathrm{RH}$ on fenofibrate in a porous silica tablet. Over a 14 day period, water was observed occupying progressively more of the porous silica matrix, decreasing the total drug signal detected, and occupying an increasing size pore space. The results indicate the potential for drug quality control by NMR relaxometry. The further development of lowfield NMR systems opens the way toward online observation during storage as well as during dissolution studies under varying conditions.

\section{AUTHOR INFORMATION}

\section{Corresponding Author}

*E-mail: jseymour@montana.edu.

ORCID

Joseph D. Seymour: 0000-0003-4264-5416

Notes

The authors declare no competing financial interest.

\section{ACKNOWLEDGMENTS}

J.D.S. acknowledges Bend, Lonza, for research funding, the U.S. NSF MRI program and Murdock Charitable Trust for equipment funding, and Dieter Gross at Bruker BioSpin for construction of the high-power probe. J.N.W. acknowledges the NSF (DMR-1455247) for research funding. The authors thank S. L. Codd for assistance.

\section{REFERENCES}

(1) Murdande, S. B.; et al. Solubility Advantage of Amorphous Pharmaceuticals: I. A Thermodynamic Analysis. J. Pharm. Sci. 2010, 99 (3), 1254-1264.

(2) Friesen, D. T.; et al. Hydroxypropyl Methylcellulose Acetate Succinate-Based Spray-Dried Dispersions: An Overview. Mol. Pharmaceutics 2008, 5 (6), 1003-1019.

(3) Wang, S. B. Ordered mesoporous materials for drug delivery. Microporous Mesoporous Mater. 2009, 117 (1-2), 1-9. 
(4) Vallet-Regi, M.; et al. A new property of MCM-41: Drug delivery system. Chem. Mater. 2001, 13 (2), 308-311.

(5) $\mathrm{Xu}, \mathrm{Z}$. P.; et al. Inorganic nanoparticles as carriers for efficient cellular delivery. Chem. Eng. Sci. 2006, 61 (3), 1027-1040.

(6) Vallet-Regi, M. Ordered mesoporous materials in the context of drug delivery systems and bone tissue engineering. Chem. - Eur. J. 2006, 12 (23), 5934-5943.

(7) Skotnicki, M.; et al. Characterization of Two Distinct Amorphous Forms of Valsartan by Solid-State NMR. Mol. Pharmaceutics 2016, 13 (1), 211-222.

(8) Pham, T. N.; et al. Analysis of Amorphous Solid Dispersions Using 2D Solid-State NMR and H-1 T-1 Relaxation Measurements. Mol. Pharmaceutics 2010, 7 (5), 1667-1691.

(9) Dempah, K. E.; Lubach, J. W.; Munson, E. J. Characterization of the Particle Size and Polydispersity of Dicumarol Using Solid-State NMR Spectroscopy. Mol. Pharmaceutics 2017, 14 (3), 856-865.

(10) Yuan, X. D.; Sperger, D.; Munson, E. J. Investigating Miscibility and Molecular Mobility of Nifedipine-PVP Amorphous Solid Dispersions Using Solid-State NMR Spectroscopy. Mol. Pharmaceutics 2014, 11 (1), 329-337.

(11) Callaghan, P. T. Translational Dynamics \& Magnetic Resonance: Principles of Pulsed Gradient Spin Echo NMR; Oxford University Press: New York, 2011.

(12) Blümich, B. NMR Imaging of Materials; Clarendon Press: Oxford, 2000.

(13) Buda, A.; Demco, D. E.; Jagadeesh, B.; Blümich, B. Molecular dynamic heterogeneity of confined lipid films by $\mathrm{H}-1$ magnetizationexchange nuclear magnetic resonance. J. Chem. Phys. 2005, 122 (3), 034701.

(14) Fujara, F.; et al. Translational and rotational diffusion in supercooled orthoterphenyl close to the glass transition. Z. Phys. B: Condens. Matter 1992, 88 (2), 195-204.

(15) Mitchell, J.; Webber, J. B. W.; Strange, J. H. Nuclear magnetic resonance cryoporometry. Phys. Rep. 2008, 461 (1), 1-36.

(16) Petrov, O. V.; Furo, I. NMR cryoporometry: Principles, applications and potential. Prog. Nucl. Magn. Reson. Spectrosc. 2009, 54 (2), 97-122.

(17) Abragam, A. Principles of Nuclear Magnetism; Oxford University Press: USA, 1983.

(18) Kubo, R. A Stochastic Theory of Line Shape and Relaxation. In Fluctuation, Relaxation, and Resonance in Magnetic Systems; Ter Haar, D., Ed.; Oliver and Boyd Ltd: Edinburgh, 1962; pp 23-68.

(19) Washburn, K. E.; et al. Simultaneous Gaussian and exponential inversion for improved analysis of shales by NMR relaxometry. $J$. Magn. Reson. 2015, 250, 7-16.

(20) Santyr, G. E.; Henkelman, R. M.; Bronskill, M. J. Variation in measured transverse relaxation in tissue resulting from spin locking with the CPMG sequence. J. Magn. Reson. 1988, 79 (1), 28-44.

(21) Song, Y. Q.; et al. T-1-T-2 correlation spectra obtained using a fast two-dimensional Laplace inversion. J. Magn. Reson. 2002, 154 (2), 261-268.

(22) Heinz, A.; et al. Understanding the solid-state forms of fenofibrate - A spectroscopic and computational study. Eur. J. Pharm. Biopharm. 2009, 71 (1), 100-108.

(23) Amstad, E.; Spaepen, F.; Weitz, D. A. Crystallization of undercooled liquid fenofibrate. Phys. Chem. Chem. Phys. 2015, 17 (44), 30158-30161.

(24) Szklarz, G.; et al. Crystallization of supercooled fenofibrate studied at ambient and elevated pressures. Phys. Chem. Chem. Phys. 2017, 19 (15), 9879-9888.

(25) Dwyer, L. M.; et al. Confined crystallization of fenofibrate in nanoporous silica. CrystEngComm 2015, 17 (41), 7922-7929. 